\title{
Combining Ability in Diallel Crosses of Quality Protein Maize Inbreds
}

\author{
D. Singh* and P. N. Jagadev \\ Department of Plant Breeding and Genetics, Odisha University of Agriculture and \\ Technology, Bhubaneswar, India \\ *Corresponding author
}

Keywords

Zea mays L., quality protein maize, combining ability, diallel cross

Article Info

Accepted:

25 March 2021

Available Online:

10 April 2021
A 5x5 diallel analysis in F1 generation including reciprocals in Quality Protein Maize (Zea mays L.) inbreds revealed that GCA and SCA effects were significant for all the traits except plant height, ear girth and number of rows per ear. Reciprocal differences were highly significant for all the characters except plant height and ear length. The ratio of GCA to SCA showed the additive and non-additive types of gene action. For most of the characters, non-additive type of gene action was predominant. In the present study, among the parents, QPM 2-18, was found to be the best general combiner having highest GCA values for most of the traits followed by QPM 8-5 and QPM 3-7 and these might be used in the hybridization programmes for combining with poor general combiner like QPM 10-1 to generate superior segregants.

\section{Introduction}

Maize (Zea mays L.), the most important cereal crop of India, assumed greater significance for food, feed and industrial utilities. Usually the hybrid development programme in maize involves the development and evaluation of inbred lines, crossing of selected inbreds and production of hybrids. Exploitation of heterosis very much depends on general combining ability (GCA) and specific combining ability (SCA) and the breeding procedure adopted. The variance due to GCA is usually considered to be an indicator of the extent of additive type of gene action and SCA is taken as the measure of non-additive type of gene action in heterosis breeding.

Hence, the present investigation was undertaken to estimate the combining ability of parents and hybrids, nature and magnitude of gene action for yield and yield components by adopting a $5 \times 5$ diallel combination of quality protein maize inbreds to increase the production and productivity. 


\section{Materials and Methods}

The experimental material comprised of five QPM inbreds (QPM 2-18, QPM 3-7, QPM 7 4, QPM 8-5 and QPM 10-1) and 20 hybrids produced by $5 \times 5$ diallel combination during rabi season. Twenty hybrids produced from 5 $\mathrm{x} 5$ full diallel combination and 5 parents were sown at EB-II Research Station of Odisha University of Agriculture and Technology, Bhubaneswar following Randomised Block Design with three replications in the following kharif season. Analysis of variance was carried out on mean values for all the 15 characters studied in F1 generation (Table 1). F1 means were used to analyse the combining ability as per Griffing's method II model (1956).

\section{Results and Discussion}

The analysis of variance revealed that both GCA and SCA variances were significant for days to $50 \%$ tasseling and silking, days to $75 \%$ dry husk, ear height, ear length, grains per row, grains per ear, grain weight, shelling $\%$, harvest index, ear weight and grain yield. (Table 1) and variance due to SCA alone was significant for plant height, ear girth and rows per ear, which indicated the importance of both additive and non-additive gene effects for most of the traits.

It was evident that the SCA variances $\left(\sigma^{2}\right.$ sca) were higher than GCA variances ( $\sigma^{2}$ gca) in all the traits and the ratios of GCA to SCA $\left(2 \sigma^{2} \mathrm{gca} / \sigma^{2} \mathrm{sca}\right)$ were less than unity, indicating the preponderance of dominance variance or non-additive type of gene action over additive type of gene action in agreement with Hassaballa et al., (1980) and Guo et al., (1986).

The GCA estimates of the parents were presented in Table 2. Parent QPM 2-18 exhibited significant positive GCA effects for all the traits except plant height, number of rows/ ear, shelling \% and harvest index. QPM 3-7 exhibited significant positive GCA effects for plant height, number of grains per ear and significant negative GCA effects for days to $75 \%$ dry husk, harvest index, ear weight/ plant and grain yield.

Parents QPM 8-5 and QPM 10-1 showed significant negative GCA effects for days to $50 \%$ tasseling and silking and $75 \%$ dry husk, ear length and grain weight. The parent QPM 2-18 proved to be the best general combiner for all the traits except shelling \% and harvest index. For grain yield and ear weight, the parents QPM 2-18 and QPM 8-5 had positive and significant GCA effects, indicating that they possessed desirable genes for increasing ear weight and grain yield.

Highest significant positive SCA effects for grain yield/ha was observed in cross QPM 8-5 x QPM 10-1, while QPM 3-7 x QPM 8-5 showed highest negative SCA for grains/ear (Table 3).

The best three crosses having high SCA for grain yield were QPM 8-5 x QPM 10-1, QPM 2-18 x QPM 8-5 and QPM 3-7 x QPM 7-4. From GCA, it was evident that parents QPM 2-18 and QPM 8-5 were the best general combiners.

Combining studies indicated that the higher contribution of SCA effects than the GCA showed the predominance of non- additive type of gene action, similar to findings of Satyanarayana et al., (1994), Shalimuddin et al., (2006) and Kanagarasu et al., (2010).

Hence, the present study revealed the importance of additive and non-additive gene effects in the inheritance of different traits. Based on GCA, the selective mating of a diallel set could be used for generating greater genetic variations to evolve better varieties. 
Table.1 ANOVA of combining ability for 15 characters in 5 x 5 fill diallel cross among 5 QPM inbreds.

\begin{tabular}{|c|c|c|c|c|c|c|c|c|c|c|c|c|c|c|c|c|}
\hline Character/source & df & $\begin{array}{c}\text { Days to } \\
50 \% \\
\text { tasseling }\end{array}$ & $\begin{array}{c}\text { Days } \\
\text { to } \\
\mathbf{5 0 \%} \\
\text { silking } \\
\end{array}$ & $\begin{array}{c}\text { Days to } \\
75 \% \\
\text { husk }\end{array}$ & $\begin{array}{c}\text { Plant } \\
\text { height } \\
(\mathrm{cm})\end{array}$ & $\begin{array}{c}\text { Ear } \\
\text { height } \\
(\mathrm{cm})\end{array}$ & $\begin{array}{c}\text { Ear } \\
\text { length } \\
(\mathrm{cm})\end{array}$ & $\begin{array}{c}\text { Ear } \\
\text { girth } \\
(\mathrm{cm})\end{array}$ & $\begin{array}{l}\text { No. Of } \\
\text { rows/ear }\end{array}$ & $\begin{array}{c}\text { No. Of } \\
\text { grains/row }\end{array}$ & $\begin{array}{l}\text { No. Of } \\
\text { grains/ear }\end{array}$ & $\begin{array}{c}100 \\
\text { grain } \\
\text { weight }(\mathrm{g})\end{array}$ & $\begin{array}{c}\text { Shelling } \\
\%\end{array}$ & $\begin{array}{c}\text { Harvest } \\
\text { index }\end{array}$ & $\begin{array}{c}\text { Ear } \\
\text { weight/plant } \\
\text { (g) }\end{array}$ & $\begin{array}{c}\text { Grain } \\
\text { yield } \\
\text { (q/ha) }\end{array}$ \\
\hline Genoytpes & 24 & $2.08 *$ & $2.11 *$ & $7.73 * *$ & $211.15^{* *}$ & $47.16 * *$ & $3.149 * *$ & $0.508 * *$ & $0.786 * *$ & $18.860 * *$ & $5010.528 * *$ & $9.754 * *$ & $0.829 * *$ & $176.194 * *$ & $694.931 * *$ & $198.482 * *$ \\
\hline GCA & 4 & $4.72 * *$ & $4.59 * *$ & $22.10 * *$ & 65.09 & $68.86 * *$ & $6.944 * *$ & 0.224 & 0.464 & $16.800 * *$ & $2792.500 * *$ & $18.281 * *$ & $0.773 *$ & $240.586^{* *}$ & $1091.078 * *$ & $297.133 * *$ \\
\hline SCA & 10 & $1.90 * *$ & $1.91 * *$ & $7.76 * *$ & $417.97 * *$ & $64.88 * *$ & $3.782 * *$ & $0.783 * *$ & $0.776 * *$ & $28.777 * *$ & $10721.550 * *$ & $11.759 * *$ & $1.120 * *$ & $149.728 * *$ & $889.473 * *$ & $257.714 * *$ \\
\hline $\begin{array}{c}\text { Reciprocal } \\
\text { effects }\end{array}$ & 10 & $1.21 * *$ & $1.31 *$ & $1.95 * *$ & 62.75 & $20.77 * *$ & 0.999 & $0.347 *$ & $0.926^{* *}$ & $9.767 * *$ & 186.664* & $4.339 * *$ & $0.558 *$ & $176.903 * *$ & $341.930 * *$ & $99.791 * *$ \\
\hline Error & 48 & 0.38 & 0.54 & 0.52 & 31.78 & 7.66 & 0.525 & 0.170 & 0.215 & 1.850 & 73.991 & 0.163 & 0.273 & 0.422 & 1.649 & 1.342 \\
\hline б2gca & & 0.25 & 0.22 & 1.42 & 0.01 & 0.01 & 0.28 & 0.01 & 0.01 & 0.01 & 0.01 & 0.69 & 0.01 & 9.76 & 24.23 & 5.04 \\
\hline$\sigma 2 \mathrm{sca}$ & & 0.90 & 0.81 & 4.31 & 229.87 & 35.06 & 1.94 & 0.37 & 0.33 & 16.03 & 6337.83 & 6.90 & 0.50 & 88.87 & 528.47 & 152.60 \\
\hline $2 \sigma 2 \mathrm{gca} / \sigma 2 \mathrm{sca}$ & & 0.55 & 0.54 & 0.66 & 0.00 & 0.00 & 0.28 & 0.05 & 0.06 & 0.00 & 0.00 & 0.20 & 0.04 & 0.22 & 0.09 & 0.07 \\
\hline h2 (ns) & & 22.98 & 20.43 & 33.88 & 0.01 & 0.01 & 17.26 & 0.01 & 0.01 & 0.01 & 0.01 & 13.13 & 0.01 & 9.90 & 6.47 & 4.72 \\
\hline
\end{tabular}

*, ** Significant at $5 \%$ and $1 \%$ levels respectively. 
Table.2 GCA effects of parents for 15 characters in F1 of a $5 \times 5$ full diallel cross among 5 QPM inbreds.

\begin{tabular}{|c|c|c|c|c|c|c|c|c|c|c|c|c|c|c|c|c|}
\hline SI.No. & Genotypes & $\begin{array}{c}\text { Days to } \\
50 \% \\
\text { tasseling }\end{array}$ & $\begin{array}{c}\text { Days } \\
\text { to } 50 \% \\
\text { silking }\end{array}$ & $\begin{array}{c}\text { Days to } \\
\mathbf{7 5 \%} \\
\text { husk }\end{array}$ & $\begin{array}{c}\text { Plant } \\
\text { height } \\
(\mathbf{c m})\end{array}$ & $\begin{array}{c}\text { Ear } \\
\text { height } \\
(\mathbf{c m})\end{array}$ & $\begin{array}{c}\text { Ear } \\
\text { length } \\
(\mathbf{c m})\end{array}$ & $\begin{array}{l}\text { Ear } \\
\text { girth } \\
(\mathrm{cm})\end{array}$ & $\begin{array}{l}\text { No. of } \\
\text { rows/ear }\end{array}$ & $\begin{array}{c}\text { No. of } \\
\text { grains/row }\end{array}$ & $\begin{array}{c}\text { No. Of } \\
\text { grains/ear }\end{array}$ & $\begin{array}{l}100 \text { grain } \\
\text { weight }(g)\end{array}$ & $\begin{array}{c}\text { Shelling } \\
\%\end{array}$ & $\begin{array}{c}\text { Harvest } \\
\text { index }\end{array}$ & $\begin{array}{c}\text { Ear } \\
\text { weight/plant } \\
\text { (g) }\end{array}$ & $\begin{array}{c}\text { Grain } \\
\text { yield } \\
\text { (q/ha) }\end{array}$ \\
\hline 1 & QPM 2-18 & $0.93 *$ & $0.88^{*}$ & $2.59 *$ & 1.39 & $4.63 *$ & $1.37 *$ & 0.24 & -0.26 & $2.00 *$ & $15.56^{*}$ & $2.39 *$ & $-0.32 *$ & $-2.57 *$ & $15.95^{*}$ & $8.06^{*}$ \\
\hline 2 & QPM 3-7 & -0.10 & -0.02 & $-0.98 *$ & $3.35^{*}$ & -0.96 & 0.02 & -0.07 & -0.11 & -0.53 & $9.19 *$ & -0.22 & 0.05 & $-2.43 *$ & $-6.15^{*}$ & $-3.20 *$ \\
\hline 3 & QPM 7-4 & $0.43 *$ & $0.45 *$ & -0.08 & -2.60 & $-1.67 *$ & -0.11 & -0.14 & -0.06 & 0.32 & 0.51 & $-0.72 *$ & $0.44 *$ & $2.07 *$ & $-1.73 *$ & -0.24 \\
\hline 4 & QPM 8-5 & $-0.53^{*}$ & $-0.59 *$ & $-0.68 *$ & -2.45 & -1.44 & $-0.45^{*}$ & 0.05 & $0.29 *$ & -0.29 & $2.72 *$ & $-0.73 *$ & -0.06 & $7.59 *$ & $3.34 *$ & $1.75^{*}$ \\
\hline 5 & QPM 10-1 & $-0.73 *$ & $-0.72 *$ & $-0.85^{*}$ & 0.31 & -0.57 & $-0.83^{*}$ & -0.08 & 0.14 & $-1.50 *$ & $-27.98^{*}$ & $-0.72 *$ & -0.10 & $-4.67 *$ & $-11.41 *$ & $-6.37 *$ \\
\hline \multicolumn{2}{|c|}{ SE(gi) } & 0.18 & 0.21 & 0.20 & 1.59 & 0.78 & 0.21 & 0.12 & 0.13 & 0.39 & 2.43 & 0.11 & 0.15 & 0.18 & 0.36 & 0.33 \\
\hline \multicolumn{2}{|c|}{ SE(gi-gj) } & 0.28 & 0.33 & 0.32 & 2.52 & 1.24 & 0.32 & 0.18 & 0.21 & 0.61 & 3.85 & 0.18 & 0.23 & 0.29 & 0.57 & 0.52 \\
\hline
\end{tabular}

Underlined figures indicate top GCA effects of the characters.

* Significant at $5 \%$ 
Table.3 SCA effects of the 10 direct crosses of 5x5 full diallel cross in QPM inbreds.

\begin{tabular}{|c|c|c|c|c|c|c|c|c|c|c|c|c|c|c|c|c|}
\hline $\begin{array}{l}\text { Sl } \\
\text { No. }\end{array}$ & Characters/ & $\begin{array}{c}\text { Days to } \\
50 \% \\
\text { tasseling }\end{array}$ & $\begin{array}{c}\text { Days } \\
\text { to } \\
50 \% \\
\text { silking }\end{array}$ & $\begin{array}{l}\text { Days } \\
\text { to } \\
75 \% \\
\text { husk }\end{array}$ & $\begin{array}{c}\text { Plant } \\
\text { height } \\
(\mathbf{c m})\end{array}$ & $\begin{array}{c}\text { Ear } \\
\text { height } \\
(\mathrm{cm})\end{array}$ & $\begin{array}{c}\text { Ear } \\
\text { length } \\
(\mathrm{cm})\end{array}$ & $\begin{array}{l}\text { Ear } \\
\text { girth } \\
(\mathrm{cm})\end{array}$ & $\begin{array}{c}\text { No. of } \\
\text { rows/ear }\end{array}$ & $\begin{array}{c}\text { No. of } \\
\text { grains/row }\end{array}$ & $\begin{array}{c}\text { No. Of } \\
\text { grains/ear }\end{array}$ & $\begin{array}{l}100 \text { grain } \\
\text { weight }(g)\end{array}$ & $\begin{array}{c}\text { Shelling } \\
\%\end{array}$ & $\begin{array}{c}\text { Harvest } \\
\text { index }\end{array}$ & $\begin{array}{c}\text { Ear } \\
\text { weight/plant } \\
\text { (g) }\end{array}$ & $\begin{array}{c}\text { Grain } \\
\text { yield } \\
\text { (q/ha) }\end{array}$ \\
\hline 1 & $\begin{array}{c}\text { QPM2- } \\
\text { 18xQPM 3- } \\
7\end{array}$ & $-0.87 *$ & $-1.18 *$ & $1.38 *$ & 6.22 & $4.90 *$ & 0.84 & 0.20 & -0.32 & $1.68 *$ & -6.13 & $1.79 *$ & -0.39 & 3.48 & $9.02 *$ & $5.49 *$ \\
\hline 2 & $\begin{array}{l}\text { QPM2-18x } \\
\text { QPM 7-4 }\end{array}$ & -0.23 & -0.31 & -0.52 & 5.64 & -0.26 & 0.49 & -0.07 & -0.24 & 0.94 & $32.66^{*}$ & $0.50 *$ & -0.09 & $1.82 *$ & $-4.09 *$ & $-1.69 *$ \\
\hline 3 & $\begin{array}{l}\text { QPM2-18X } \\
\text { QPM8-5 }\end{array}$ & $-0.90^{*}$ & $1.22 *$ & $0.91 *$ & 1.66 & $5.91 *$ & $1.19 *$ & $0.67 *$ & $0.95^{*}$ & $4.84 *$ & $113.85^{*}$ & $1.36^{*}$ & -0.05 & 0.37 & $16.68 *$ & $9.83 *$ \\
\hline 4 & $\begin{array}{c}\text { QPM 2-18X } \\
\text { QPM10-1 }\end{array}$ & $-0.73^{*}$ & -0.65 & 0.08 & 5.49 & -0.93 & -0.58 & $0.60 *$ & 0.50 & $-1.72 *$ & $-12.59 *$ & 0.26 & -0.51 & $4.09 *$ & 1.00 & 0.62 \\
\hline 5 & $\begin{array}{l}\text { QPM3-7 X } \\
\text { QPM 7-4 }\end{array}$ & -0.70 & -0.41 & -0.12 & $10.74 *$ & 1.60 & 0.22 & 0.02 & 0.49 & -0.60 & $25.49 *$ & 0.23 & $0.73 *$ & $6.62 *$ & $16.45^{*}$ & $8.57 *$ \\
\hline 6 & $\begin{array}{c}\text { QPM 3-7 X } \\
\text { QPM 8-5 }\end{array}$ & $1.43 *$ & $1.29 *$ & $\begin{array}{c}- \\
1.19 *\end{array}$ & $8.33^{*}$ & 0.55 & -0.48 & -0.01 & -0.26 & $-2.72 *$ & $-34.19 *$ & -0.16 & -0.05 & $1.44 *$ & $-15.06^{*}$ & $-7.11 *$ \\
\hline 7 & $\begin{array}{l}\text { QPM 3-7 X } \\
\text { QPM 10-1 }\end{array}$ & $0.80^{*}$ & 0.59 & ${ }^{-}$ & 1.59 & -0.01 & -0.71 & -0.30 & $0.82 *$ & 0.18 & $62.95 *$ & $-1.39 *$ & -0.47 & $-1.31 *$ & $-18.20^{*}$ & $-8.82 *$ \\
\hline 8 & $\begin{array}{l}\text { QPM 7-4 X } \\
\text { QPM 8-5 }\end{array}$ & 0.07 & -0.01 & $1.91 *$ & 3.21 & 0.78 & 0.16 & 0.29 & 0.02 & $2.40 *$ & $25.43 *$ & $1.36^{*}$ & $0.68 *$ & $-14.48 *$ & $9.77 *$ & $6.20 *$ \\
\hline 9 & $\begin{array}{l}\text { QPM 7-4 X } \\
\text { QPM 10-1 }\end{array}$ & -0.40 & -0.38 & $2.25 *$ & 0.18 & $3.71 *$ & $1.45^{*}$ & 0.44 & 0.17 & $2.30 *$ & $27.86^{*}$ & $1.55^{*}$ & $0.79 *$ & $-5.85^{*}$ & $3.50 *$ & $2.28 *$ \\
\hline \multirow[t]{3}{*}{10} & $\begin{array}{l}\text { QPM 8-5 X } \\
\text { QPM 10-1 }\end{array}$ & -0.26 & -0.68 & $2.01 *$ & $17.13^{*}$ & $5.91 *$ & $1.81 *$ & 0.31 & -0.38 & $4.04 *$ & $32.49^{*}$ & $3.16^{*}$ & 0.34 & $13.94 *$ & $34.94 *$ & $17.08 *$ \\
\hline & SE(ij) & 0.36 & 0.43 & 0.42 & 3.29 & 1.61 & 0.42 & 0.24 & 0.27 & 0.80 & 5.02 & 0.24 & 0.31 & 0.38 & 0.75 & 0.68 \\
\hline & SE(Sij-Skl) & 0.48 & 0.57 & 0.56 & 4.37 & 2.14 & 0.56 & 0.32 & 0.36 & 1.05 & 6.66 & 0.31 & 0.41 & 0.50 & 1.00 & 0.90 \\
\hline
\end{tabular}

* Significant at $5 \%$ level

The SCA effects of the reciprocal crosses were the same as direct crosses.

Underlined figures indicated the highest SCA effects for each character. 


\section{References}

Griffing, B. 1956. Concept of general and specific combining ability in relation to diallel crossing system. Australian J. Biol. Sci. 9: 463-493.

Guo, P. Z., Gardener, C. O. and Obaidi, M. 1986. Genetic variations and gene effects controlling prolificacy and other traits in maize (Zea mays L.). Acta Genetica Sinica. 13: 35-42.

Hassaballa, E. S., El- Morshidy, M. A., Kalifa, M. A. and Shalaby, E.M. 1980. Combining ability analysis in maize. Flowering Res. Bull., Faculty of Agric., Ain Shams Univ., Egypt. 1291:
8.

Kanagarasu, S., Nallathambi, G. and Ganesan, K.N. 2010. Combining ability analysis for yield and its component traits in maize (Zea mays L.). e-J. Plant Breed. 4: 915-920.

Satyanarayana, E., Kumar, R. S. and Sharma, M.Y. 1994. Inheritance studies of maturity components and yield in selected hybrids of maize (Zea mays L.). Mysore J. Agric. Sci. 28: 25-30.

Shalimuddin, M., Khatun, F., Ahmed, S., Ali, M.R. and Bagum, S.A. 2006. Heterosis and combining ability in corn (Zea mays L.). Bangladesh J. Bot. 35: 109116.

\section{How to cite this article:}

Singh, D. and Jagadev, P. N. 2021. Combining Ability in Diallel Crosses of Quality Protein Maize Inbreds. Int.J.Curr.Microbiol.App.Sci. 10(04): 894-899.

doi: https://doi.org/10.20546/ijcmas.2021.1004.095 